\title{
Constipação intestinal em crianças e a importância das fibras alimentares: Uma revisão da literatura
}

\author{
Intestinal constipation in children and the importance of dietary fiber: A review of the \\ literature
}

Constipación intestinal en niños y la importancia de las fibras alimentarias: Una revisión de la literatura

\begin{abstract}
Vanessa Bezerra Borges de Sousa ${ }^{1}$, Larruama Priscylla Fernandes de Vasconcelos ${ }^{1 *}$, Débora Gomes de Sousa Araújo ${ }^{1}$, Jullyane de Oliveira Maia Lemos ${ }^{2,3}$, Luiza Satyro Morais de Medeiros ${ }^{1}$, Raquel Bezerra de Sá de Sousa Nogueira ${ }^{1}$, Claudio Leite da Silva ${ }^{1}$, Francisca Mamede Leite ${ }^{1}$, Paloma Cyntia da Silva Figueiredo Siqueira ${ }^{1}$, Erika Epaminondas de Sousa ${ }^{1}$, Alcides Pereira Lino Júnior ${ }^{1}$.
\end{abstract}

\section{RESUMO}

Objetivo: Observar a relação entre a constipação intestinal em crianças e a importância do consumo regular de fibras alimentares na sua prevenção. Métodos: Tratou-se de uma revisão da literatura, de abordagem qualitativa dos dados e caráter descritivo, onde o processo de formulação se deu através de buscas por literaturas científicas, em base de dados, tendo como descritores: Constipação intestinal infantil, consumo alimentar e fibras alimentares. Resultados: A alimentação exerce grande papel na prevenção e/ou tratamento da constipação intestinal, sendo esta composta por frutas, verduras e cereais integrais, alimentos esses que tem um adequtado aporte de fibras, que são os nutrientes mais importantes na constipação intestinal. Além das fibras, outros componentes como os prebióticos que são encontrados em alimentos de origem vegetal, tem grande papel nessa patologia, uma vez que uma vez que a alteração da microbiota também está associada à constipação intestinal, pois um possível desequilíbrio de bactérias pode afetar o trânsito intestinal e ter como consequência a constipação. É necessário também uma ingestão hídrica adequada, pois está auxilia na eliminação do bolo fecal. Considerações Finais: A constipação intestinal é um problema frequente na infância, e alimentação inadequada, pobre em fibras e rica em carboidratos é a maior causa desse problema, sendo assim os hábitos alimentares saudáveis são necessários para o combate desse problema.

Palavras-Chaves: Constipação intestinal, Consumo Alimentar, Fibras alimentares.

\begin{abstract}
Objective: Observe the relationship between intestinal constipation in children and the importance of regular consumption of dietary fiber in its prevention. Methods: This was a review of the literature, a qualitative approach to the data and a descriptive character, where the formulation process was carried out through searches of scientific literature, based on data, having as descriptors: Constipation, food consumption and fibers food. Results: Feeding plays a major role in the prevention and / or treatment of intestinal constipation, which consists of fruits, vegetables and whole grains, which have the adequate supply of fiber, which are the most important nutrients in intestinal constipation. In addition to the fibers, other components such as prebiotics that are found in foods of plant origin play a major role in this pathology, since since the microbiota alteration
\end{abstract}

\footnotetext{
${ }^{1}$ Faculdades Integradas de Patos (FIP). Patos-PB

2 Faculdade de Ciências Médicas de Campina Grande-PB. Campina Grande-PB

*E-mail: larruama priscylla@hotmail.com
}

SUBMETIDO EM: 10/2018

ACEITO EM: 11/2018

PUBLICADO EM: 03/2019

REAS/EJCH | Vol.Sup.21 | e561 |DOI: https://doi.org/10.25248/reas.e561.2019 Página 1 de 9 
is also associated with intestinal constipation, a possible disequilibrium of bacteria can affect the intestinal transit and result in constipation. Adequate water intake is also necessary as it helps to eliminate fecal cakes. Final conclusion: Intestinal constipation is a common childhood problem, and inadequate, low-fiber, highcarbohydrate diet is the major cause of this problem, so healthy eating habits are needed to combat this problem.

Key Words: Constipation, Food Consumption, Food fibers.

\section{RESUMEN}

Objetivo: Observar la relación entre el estreñimiento intestinal en los niños y la importancia del consumo regular de fibras alimentarias en su prevención. Métodos: Se trató de una revisión de la literatura, de abordaje cualitativo de los datos y carácter descriptivo, donde el proceso de formulación se dio a través de búsquedas por literaturas científicas, en base de datos, teniendo como descriptores: Constipación intestinal, consumo alimentario y fibras alimentarias. Resultados: En la mayoría de los casos, la mayoría de las personas que sufren de depresión en el intestino delgado, que tienen la grasa de la fibra, que tienen la calidad de los alimentos en el intestinal constipation. Además de las fibras, otros componentes como las predicciones que se encuentran en el alimento de la planta originan el rol principal en esta patología, debido a que la microbiota alteración está también asociada con el intestinal constipation, el posible desbalance de las bacterias afectan el intestinal transit y resultado en constipation. Adecuación de agua de agua también es necesaria para eliminar fecal cakes. Consideraciones finales: Intestinal constipation es un problema complejo, e inadecuado, low-fiber, high-carbohidratediet es el mayor causa de este problema, son saludables alimentos son necesarios para combatir este problema.

Palabras clave: Estreñimiento intestinal, Consumo Alimentario, Fibras alimenticias.

\section{INTRODUÇÃO}

A constipação intestinal vem se tornando um problema muito comum no público infantil, sendo um dos distúrbios intestinais mais comuns nessa população (FERREIRA JF et al., 2013). Nas crianças, a constipação crônica está entre os dez problemas mais comuns na prática pediátrica geral (CLAYDEN GS et al., 2005). Apesar de não apresentar risco a vida, proporciona aos indivíduos acometidos, especialmente quando são crianças, um grande desconforto e baixa na qualidade de vida (MACHADO WM e CAPELARI SM, 2010; GOMES PB et al., 2011).

Dentre os diversos tipos de constipação intestinal, destaca-se a do tipo funcional, sendo esta a mais comum, até mesmo no público infantil, tendo em vista que a maioria das crianças apresentam constipação funcional, e ao que se indica, o referido problema se inicia no desmame com a introdução de alimentos sólidos, com baixo teor de fibras e ingestão hídrica insuficiente (BELO GMS et al., 2008; GOMES PB et al., 2011; GAVANSKI DS et al., 2015).

Cardoso AL (2013) complementa que existem três períodos em que as crianças em crescimento estão mais sujeitas a apresentar este quadro de constipação: após a introdução de alimentos sólidos e cereais; na época do treinamento de esfíncteres e quando a criança começa a frequentar a escola.

A constipação intestinal é, na maioria das vezes, atribuída ao trânsito retossigmóide prolongado, resultado de uma ingestão insuficiente de líquidos, fibras alimentares e sedentarismo. A do tipo funcional caracterizase pela ausência de causas orgânicas, detectáveis pelos métodos de investigações atualmente disponíveis (GAVANSKI DS et al., 2015).

As fibras alimentares são encontradas nos alimentos de origem vegetal, constituem a parte que o organismo é incapaz de digerir e embora não forneça nutrientes ao organismo, sua falta acarreta prejuízos à saúde, pois ela tem a capacidade de absorver líquidos e aumentar a matéria do bolo fecal, sendo indispensável no tratamento de doenças do trato gastrointestinal, como a constipação intestinal (BODINSKI 
$\mathrm{CH}, 2006)$. São compostas de celulose, hemicelulose, gomas, pectinas, lignina, materiais ricos em amido e oligossacarídeos e pode ser fornecida através da ingestão de alimentos como frutas, verduras, grãos integrais, legumes, nozes e sementes, que além de fibras, também são ricos em prebióticos (MAHAN LK et al., 2012).

As fibras são classificadas em solúveis e insolúveis, de acordo com seu grau de solubilidade com a água, sendo a primeira solúvel em água e a subsequente não solúvel em água, onde as fibras insolúveis agem melhorando o trânsito intestinal e as fibras solúveis, retardando o esvaziamento gástrico, além de ser de suma importância para a redução dos níveis de glicemia e colesterol (PAULA LD et al., 2009; MIRA GS et al., 2009).

Os prebióticos podem ser conceituados como todo ingrediente alimentar não digerível que afeta de maneira benéfica o organismo por estimular o crescimento e/ou atividade da microbiota do cólon, fazendo prevalecer às colônias benéficas. Podem ser classificados como fibras, porém nem toda fibra constitui um prebióticos (STEFE CA et al., 2008; BROWNAWELL AM et al., 2012).

De acordo com Cardoso AL (2013), o tratamento da constipação intestinal consiste em meios medicamentosos via oral ou fecal, na mudança dos hábitos alimentares, como aumento do consumo de fibras, como vegetais crus, frutas e grãos e cereais, e de líquidos; na observação/identificação dos sintomas e impactação das fezes; e na educação nutricional dos pais e das crianças, através da conscientização da importância da adesão ao tratamento e da importância da alimentação adequada.

O sucesso do tratamento virá principalmente com as mudanças do estilo de vida. Os alimentos industrializados devem ser substituídos por alimentos naturais e ricos em fibras, aliado a uma hidratação adequada e prática regular de exercício físico (VITOLO MR, 2007).

Dessa forma, objetivou-se observar a relação entre a constipação intestinal, que acomete em exponencial o público infantil, especialmente devido aos hábitos alimentares inadequados que vem sendo impostas a esse público, e o consumo regular de fibras alimentares.

\section{METODOLOGIA}

Tratou-se de uma revisão da literatura, de abordagem qualitativa dos dados e caráter descritivo, onde o processo de formulação se deu através de buscas por literaturas científicas, em base de dados como Scientific Electronic Library Online (SCIELO), National Library of Medicina National (PUBMED), Literatura Latinoamericanas e do Caribe (LILACS), bem como na base de dados do google acadêmico, nas línguas portuguesa e inglesa, tendo como descritores: Constipação intestinal, fibras alimentares, crianças.

Foram incluídos na pesquisa todos os artigos que apresentaram temática central do estudo dos últimos 15 anos, dando preferência as mais atuais e excluídos todos aqueles que não contribuiriam positivamente para o estudo e/ou não apresentaram a temática central da pesquisa.

\section{RESULTADOS E DISCUSSÃO}

De acordo com Alves JG (2013) a constipação intestinal pode ser dividida em duas categorias: a de trânsito normal ou funcional e a constipação de trânsito lento, sendo a primeira mais comum e geralmente é ocasionada pela insuficiência de fibras e água na dieta e respondem a adição destes componentes à dieta de forma mais rápida, em relação a constipação do trânsito lento. Nela, o indivíduo costuma evacuar cerca de uma vez por semana, com desconfortos abdominais e é mais comum em mulheres jovens.

A constipação do tipo funcional, mais comum nas crianças, persiste em evacuações dolorosas, e persistentemente difíceis, pouco frequentes e quase todas as vezes incompletas (CARDOSO AL, 2013). A 
fisiopatologia da doença é complexa e multifatorial e resultada da combinação direta ou indireta de fatores estruturais, metabólicos, anatômicos, mecânicos, e funcionais sobre o cólon, reto e ânus (TRISÓGLIO C et al., 2010).

A causa da constipação infantil é multifatorial. Fatores genéticos, alterações na motilidade gastrointestinal, retenção fecal, hábitos alimentares, aspectos psicossociais e história familiar são fatores relacionados com o surgimento e a manutenção da constipação infantil (SPERIDIAO PDGL et al., 2003; INAN M et al., 2007).

Os fatores de risco para a constipação são: prematuridade, atraso de eliminação de mecônio, alteração do desenvolvimento neuropsicológico, atraso no desenvolvimento, obesidade, hipotireoidismo, apetite seletivo, uso de medicamentos constipantes - antidepressivos, anticonvulsivantes, anti-histamínicos, diuréticos, opiáceos, antiácidos, antidiarreicos, suplementos de cálcio e ferro e anti-inflamatórios não esteróides, baixo consumo de fibras, ingestão de leite em excesso (GIBAS-DORNA M e PIATEK J, 2014; THARNER A et al., 2015). Além de condições inadequadas para a evacuação, abuso sexual e história familiar de constipação, ansiedade e/ou depressão (INALOO S et al., 2014).

A má alimentação é uma das principais causas da constipação intestinal, sendo uma característica predominante no hábito alimentar de indivíduos que sofrem dessa anomalia. Observa-se, nesses indivíduos, o consumo de alimentos industrializados e refinados, ocasionando incapacidade progressiva do intestino grosso deslocar os resíduos fecais para sua expulsão (BELO GMS et al., 2008; GOMES PB et al., 2011).

No estudo realizado por Mello CS et al. (2010), evidenciou-se relação direta entre o consumo de fibras e a constipação, onde uma dieta rica em fibras faz com que melhore este quadro, assim como no consumo insuficiente destes há presença de constipação e excesso de peso. Isto também é evidenciado na pesquisa de Machado WM e Capelari SM (2010), onde a utilização das fibras para indivíduos com problemas intestinais mostrou efeitos benéficos em $64 \%$ dos casos. Isso acontece, pois as fibras formam uma massa fecal que atrai água para a luz intestinal, tendo em vista que a constipação intestinal está diretamente ligada com o tempo de trânsito do material fecal, visto que quanto mais tempo as fezes permanecem no cólon e reto, mais água é retida e mais duras as fezes se tornam (BODINSKI CH, 2006).

Em um ensaio clínico realizado por Castillejo G (2006) com crianças de 3 a 10 anos, observou-se melhora no trânsito intestinal, bem como melhora da consistência das fezes, quando comparado ao grupo que não recebeu o suplemento de casca de cacau rico em fibra.

Fagundes e Costa (2003 apud SANT'ANNA MSL, FERREIRA CLLF, 2016) afirmam que a importância da utilização das fibras alimentares foi reconhecida há mais de vinte anos, através de estudos que evidenciavam que o consumo destas poderiam prevenir diversas doenças como constipação intestinal, hemorróidas, aterosclerose, câncer de cólon, entre outras doenças, inteiramente ligadas ao consumo de alimentos pobres em fibras e refinados.

Quanto ao tipo de fibras, as fibras insolúveis são as que exercem papel de prevenção e/ou tratamento da constipação intestinal, pois elas fazem com que haja um aumento do bolo fecal e à redução de trânsito intestinal, retardando o esvaziamento gástrico e o trânsito intestinal, já as fibras solúveis em água ajudam a manter os níveis de glicose no sangue, além de reduzir os níveis de colesterol (MORRIS DH, 2007; PAPATHANASOPOULOS A e CAMILLERI M, 2010). Isto é evidenciado no estudo de Paula LD et al. (2009), com camundongos, onde tais autores observaram que as fibras insolúveis, aliada a uma dieta balanceada apresentou uma eliminação de fezes relativamente maior que as solúveis, presentes no "mucilon" de arroz e de milho, pois essas estimulam os nervos da parede intestinal, melhorando a regulação do intestino.

Papathanasopoulos A e Camilleri M (2010) ainda afirmam que fibras solúveis, como as pectinas, mucilagens, gomas e algumas hemiceluloses podem ser encontradas frutas, verduras, aveia, cevada, leguminosas como feijão, lentilha e grão de bico. Já as fibras insolúveis, como a como a celulose, lignina e algumas hemiceluloses, têm como fontes principais o farelo de trigo, grãos integrais e verduras. Segundo 
Escott-Stump S (2011) para cada grama de fibra de cereais, o peso das fezes aumenta em torno de 3 a 9 gramas.

O Comitê de Nutrição da Academia Americana de Pediatria recomenda que a quantidade de fibra alimentar seja de 0,5g/Kg/dia (BIGÉLLI RHM et al., 2004). A partir da adolescência, Bernaud PSR e Rodrigues TC (2013) preconizam que a ingestão média de fibras na alimentação deve ser em torno de 30 gramas por dia, através de alimentos variados ricos em fibras como frutas, verduras, farelos e grãos integrais, para que os benefícios possam ser alcançados, como melhora do quadro de constipação intestinal e redução dos níveis de glicose, pressão arterial e lipídios séricos.

Entretanto, grande parte da população não consume a quantidade ideal de fibras na dieta. No estudo de Martinez-Costa C et al. (2005), realizado com 62 crianças portadoras de constipação intestinal, observou-se que grande parte da amostra (60\%) apresentaram uma ingestão deficiente de fibras alimentares. Além disso, no estudo de Maffei HVL (2011), foi encontrado resultados significativos relativo a melhora dos sintomas de constipação após a inserção de dieta rica em grãos em 28 crianças constipadas.

O baixo consumo de verduras e frutas é um fator preocupante, já que são alimentos fontes de fibras, importantes na regulação da função intestinal. O consumo de frutas e verduras na infância é muito importante, pois esses alimentos são excelentes fontes de vitaminas, minerais e fibras, atuando na prevenção e correção da desnutrição, deficiência de vitamina A, ferro e zinco (OSORIO MM e FARIAS JUNIOR G, 2005; BARBOSA RMS, 2006).

A alimentação da criança constipada deve ser rica em frutas, principalmente aquela de efeito laxante, como mamão, ameixa, laranja com bagaço, abacate, abacaxi, tangerina e melancia. A banana e a maçã, como não possuem muito efeito sobre a função intestinal, podem ser consumidas com aveia ou farelo (ACCIOLY E et al., 2009).

Além das frutas, devem ser oferecidos às crianças diariamente legumes e vegetais folhosos como alface, espinafre, couve, bertalha, brócolis, agrião, de preferência cru e o uso de cereais e pães integrais deve ser incentivado, mesmo aqueles pouco estimulantes do peristaltismo intestinal, como pão branco, arroz, batata, uma vez que podem ser usados em combinação com alimentos fonte de fibras, como por exemplo, arroz cozido com folhosos (ACCIOLY E et al., 2009).

Schröeder e Santos (2009) sugerem que um alimento funcional de extrema importância para a melhora significativa da constipação intestinal é a semente de linhaça, se utilizada na quantidade e maneira correta. Um terço da fibra dietética total desse alimento é solúvel e todo o restante constitui-se em fibras insoluvéis e além das fibras, contêm componentes como os compostos fitoquímicos e o ácido linolêico que além de ajudar atuar diretamente na prevenção e/ou tratamento da constipação intestinal (MORRIS DH, 2007; CUPERSMID L et al., 2012).

Ainda assim, Mahan LK et al. (2012) relatam a necessidade da importância de não ingerir as fibras em excesso, pois nesse caso, pode haver uma distensão abdominal e resultar em flatulência excessiva. Além disso, segundo Galisa MS et al. (2008), seu excesso pode interferir na absorção de cálcio e zinco, principalmente no público infantil e idoso.

Os prebióticos acarretam benéficos na regulação do hábito intestinal, pois a microbiota intestinal está relacionada à constipação intestinal. Quando há um desequilíbrio das bactérias, o trânsito intestinal é afetado. (CARVALHO FL, 2016). Khalif IL et al. (2005) detectaram que pacientes constipados possuem em sua microbiota intestinal um menor número de bactérias do gênero Bifidobacterium e Lactobacillus quando comparados a pacientes sem constipação intestinal.

Para uma substância, ou grupo de substâncias, ser definida como prebiótico, ela deve obedecer alguns requisitos: ter origem vegetal; ser parte de um conjunto heterogêneo de moléculas complexas; não ser 
hidrolisada por enzimas digestivas; não ser absorvida na parte superior do trato gastrintestinal; ser parcialmente fermentável por colônias de bactérias e ser osmoticamente ativa. (MOROTI C et al., 2009).

Tradicionalmente, os prebióticos estão presentes em verduras e frutas como cebola, banana, alho e alcachofra e sob a forma de oligossacarídeos no leite materno (KAUR N e GUPTA GA, 2002; FAN W et al., 2014; GARRIDO D et al., 2013).

O prebióticos são fermentados no colón pelas bactérias anaeróbicas onde estas produzem o ácido lático, ácidos graxos de cadeia curta (AGCC) como o acetato, butirato e propinato, reduzindo o $\mathrm{pH}$ no lúmen intestinal, estímulo da proliferação de células epiteliais do cólon, incremento da motilidade colônica, aumento da frequência das evacuações e aumento do volume das fezes. Dessa forma, a ação do probiótico na constipação ocorre quando esses carboidratos não sofrem ação enzimática no trato gastrointestinal (BELELI CAV, 2013).

A presença de fibras também estimula o crescimento de gêneros bacterianos como Bifidobacterium e Lactobacillus, atuantes na motilidade intestinal devido as suas atividades fermentativas e sacarolítica (PAYNE NA et al., 2012; SHEN Q et al., 2011) Estudos realizados com as bactérias do gênero Bifidobacterium demonstraram uma redução de até $38 \%$ do tempo de trânsito intestinal em pacientes com trânsito intestinal lento e de até $42 \%$ naqueles com trânsito intestinal normal (BOUVIER M et al., 2001; MARTEAU P et al., 2002).

Ao avaliar os efeitos in vitro das fibras na composição da microbiota fecal, através de análises moleculares, foi verificado que pectina e amido resistente promovem o crescimento de bifidobactérias (YANG SD et al., 2013). Quanto a resposta da microbiota intestinal infantil, De Fillipo $C$ et al. (2010) relataram que crianças que apresentam uma dieta rica em fibras possuem em sua um número maior de bifidobactérias quando comparadas àquelas que consomem uma dieta com baixo teor desse nutriente (DE FILLIPO C et al., 2010). No seu estudo, Bernal MJ et al. (2013) observaram resultados semelhantes.

Os estudos com seres humanos mostram que uma alimentação rica em carboidratos não-digeríveis como prebióticos e oligossacarídeos não digeríveis estimula a microbiota intestinal e a bifidogênese, fatores importantes no tratamento e prevenção da constipação intestinal. Enquanto que uma alimentação pobre em fibras e rica em açúcares simples, proteínas, amidos e lipídeos provocam efeitos deletérios na microbiota intestinal (MARTINEZ-COSTA C et al., 2005; DE FILIPPO C et al., 2010; SHEN Q et al., 2012).

Machado WM (2010) preconiza que a ingestão hídrica também apresenta papel indispensável, quando aliado a ingestão de fibras, para auxiliar na ação terapêutica desta, tendo em vista que sem a quantidade adequada de água de líquido elas não funcionam satisfatoriamente. Além disso, a hidratação, auxilia na eliminação dos bolos fecais, especialmente recessados.

De acordo com Cardoso AL (2013) a recomendação é de que a criança ingira cerca de 1 a 1,5 litros de água ou outro líquido que não seja leite, principalmente quando as fibras estiverem presentes na dieta.

Segundo Pereira VP et al. (2009), o comportamento da criança durante as refeições, assim como suas preferências alimentares, geralmente reflete os hábitos alimentares de sua família, de acordo com as crenças, significados, condições socioeconômicas e valores ligados à alimentação. Em seu estudo, eles constataram que o fator financeiro é limitante para definir os alimentos que compõem a dieta dessas crianças e que apesar de grande parte de mães de crianças com constipação intestinal crônica saberem que a alimentação é importante no tratamento dessa doença, apenas uma parte tem conhecimento sobre papel das fibras alimentares para o tratamento e/ou prevenção da constipação intestinal.

O reconhecimento da necessidade das práticas alimentares deve incluir o questionamento sobre a forma que a família organiza o dia alimentar, como quais os alimentos serão disponibilizados, quem preparará as refeições da família, com que frequência se utilizam bebidas prontas com açúcar, salgados, a utilização do 
açúcar e em quais preparações, com que frequência se utilizada molhos ou alimentos prontos, onde são realizadas as refeições, em que horários, dentre outros (MONTEIRO JP e CAMELO JÚNIOR JS, 2011).

A melhora e/ou reversão do quadro constipação, especialmente a funcional crônica, depende de fatores fundamentais para o alcance de bons resultados, tais como a adesão da criança ao tratamento (ingestão da dieta com quantidade adequada de fibras, medidas comportamentais, desimpactação de fezes e terapêutica medicamentosa) e entendimento dos pais sobre a importância e necessidade do tratamento e das mudanças nos hábitos alimentares da criança (CRUZ DO, 2010).

\section{CONSIDERAÇÕES FINAIS}

A constipação intestinal é um problema comum em crianças, e os fatores para seu desenvolvimento são hábitos de vida e alimentares inadequados. Sendo assim, são necessários mudanças na alimentação como a inclusão de alimentos com maior aporte de fibras alimentares, pois estes são os nutrientes necessários na regulação do transito intestinal, tanto as fibras solúveis e insolúveis devem ser incluídas na alimentação.

Além disso, a regulação da microbiota intestinal através do uso de prebióticos também é necessária, uma vez que o desequilíbrio da flora intestinal pode levar a constipação. É necessário incluir na alimentação frutas, verduras, e cereais integrais e diminuir o consumo de carboidratos simples. O adequado aporte hídrico também é importante, pois está diretamente relacionada com os benefícios das fibras, formando géis e conseguintemente melhorando a eliminação do bolo fecal.

Ressalta-se que a família tem grande papel no tratamento e/ou prevenção dessa patologia, pois todos os que convivem com a criança, são um espelho para adesão de hábitos adequados e inadequados. Sendo assim, é necessário que seja feita uma educação alimentar com pais e responsáveis pela criança.

\section{REFERÊNCIAS}

1. ACCIOLY E, SAUNDERS C, LACERDA E. Nutrição em obstetrícia e pediatria. São Paulo: Cultura Médica, 2009.

2. ALVES JG. Constipação Intestinal. Jornal Bras. de Medicina, 2013; 101 (2): 31-37.

3. BARBOSA RMS. Avaliação do consumo alimentar de crianças pertencentes a uma creche filantrópica na ilha de Paquetá, Rio de Janeiro, Brasil. Revista Brasileira de Saúde Materno Infantil. 2006; 6(1):127-134.

4. BELELI CAV. Efeito do prebiótico 4' galactooligossacarídeo em crianças e adolescentes com constipação funcional. 2014. [Dissertação]. Campinas: Faculdade de Ciencias Medicas; 2014.

5. BELO GMS, DINIZ AS, PEREIRA APC. Efeito terapêutico da fibra goma-guar parcialmente hidrolisada na constipação intestinal funcional em pacientes hospitalizados. Arq. Degastroenterologia, 2008; 45 (1): 93-95.

6. BERNAL MJ, PERIAGO MJ, MARTÍNEZ R et al. Effects of infant cereals with different carbohydrate profiles on colonic function randomised and double-blind clinical trial in infants aged between 6 and 12 months-- pilot study. EuropeanJournalofPediatrics, 2013; 172 (11): 1535-1542.

7. BERNAUD FSR, RODRIGUES TC. Fibra alimentar- Ingestão adequada e efeitos sobre a saúde do metabolismo. Arq. Bras. Endocrinol. Metab., 2013; 57 (6): 397-405.

8. BIGÉLLI RHM, FERNANDES MIM, GALVÃO LC. Constipação intestinal na criança. Rev. de Medicina. 2004; 37(1): 65-75.

9. BODINSKI LH. Dietoterapia: princípios e práticas. São Paulo: Editora Atheneu, 2006.

10. BOUVIER M, MEANCE S, BOULEY $C$ et al. Effects of consumption of a milk fermented by the probiotic Bifidobacteriumanimalis DN 173010 on colonic transit time in healthy humans. Biosci Microflora, 2001; 20: 4348.

11. BROWNAWELL AM, CAERS W, GIBSON GR et al. Prebiotics and the Health Benefits of Fiber: Current Regulatory Status , Future Research , and Goals 1 , 2. The JournalofNutrition, 2012; 142:962-974.

12. CARDOSO AL. Constipação e cólicas na infância: causas e manejo terapêutico. Pediatr. Mod., 2013; 49, (4):139148. 
13. CARVALHO FL. Prevalência de constipação intestinal e fatores associados aos seis meses de idade. [Dissertação]. Feira de Santana: Universidade Estadual de Feira de Santana, 2016.

14. CASTILLEJO, G. et al. A controlled, randomized, double-blind trial to evaluate the effect of a supplement of cocoal husk that is rich in dietary fiber on cocoa husk that is rich in dietary fiber on colonic transit in constipated pediatric patients. Pediatrics. 2006; 118(3):641-8

15. CLAYDEN GS, KESHTGAR AS, CARCANI-RATHWELL I et al. The management of chronic constipation and related faecal incontinence in childhood. Arch Dis Child Educ Pract Ed., 2005; 90(3). 58-67.

16. CRUZ DO, NEUFELD CB, TOPOROVSKI MS. Manometria anorretal em crianças com constipação intestinal crônica funcional refratária a tratamento. Rev. Paul. Pediatr.,2010; 28 (4): 347-51.

17. DE FILIPPO C, CAVALIERI D, DI PAOLA M et al. Impact of diet in shaping gut microbiota revealed by a comparative study in children from Europe and rural Africa. Proceedings of the National Academy of Sciences of the United States of America, 2010; 107(33): 14691-6.

18. FAN W, HUO G, LI X et al. Impact of diet in shaping gut microbiota revealed by a comparative study in infants during the six months of life. Journal of Microbiology and Biotechnology, 2014; 24(2): 133-43.

19. FERREIRA JF, SILVA DCG, NASSIF CAM et al. Hábitos alimentares e ocorrência de constipação intestinal em crianças de 3 a 6 anos de uma escola pública do município de Itaperuna (RJ). Rev. Cient. da Faminas, 2013; 9(2): 70-84.

20. GALISA MS, ESPERANÇA LMB, SÁ NG. Nutrição: Conceitos e aplicações. São Paulo: M. Books do Brasil Editora Ltda, 2008.

21. GARRIDO D, DALLAS DC, MILLS DA. Consumption of human milk glycoconjugates by infant-associated bifidobacteria: mechanisms and implications. Microbiology. 2013; 159(4): 649-64.

22. GAVANSKI DS, BARATTO I, GATTI RR. Avaliação do hábito instestinal e ingestão de fibras alimentares em uma população de idosos. Rev. Bras de Obesidade, Nutrição e Emagrecimento, 2015; 9(49): 3-11.

23. GIBAS-DORNA M, PIATEK J. Functional constipation in children: evaluation and management. Prz Gastroenterol., 2014; 9(4):194-9.

24. GOMES PB, MELO MCB, DUARTE MA et al. Polietilenoglicol na constipação intestinal crônica funcional em crianças. Rev. Paul. Pediatr., 2011; 29(2): 245-50.

25. GOMES PB, MELO MDCB, DUARTE MA et al. Polietilenoglicol na constipação intestinal crônica funcional em crianças. Revista Paulista de Pediatria., 2011; 2(29):245-50.

26. INALOO S, DEHGHANI SM, HASHEMI SM et al. Comorbidity of headache and functional constipation in children: a crosssectional survey. Turk J Gastroenterol, 2014; 25(5):508-11.

27. INAN M, AYDINER CY, TOKUC B et al. Factors associated with childhood constipation. J Paediatr Child Health, 2007; 43(10):700-6.

28. KAUR N, GUPTA GA. Review Applications of inulin and oligofructose in health and nutrition. J Biosci., 2002; 27(7): 703-714.

29. KHALIF IL, QUIGLEY EMM, KONOVITCH EA et al. Alterations in the colonic flora and intestinal permeability and evidence ofimmune activation in chronic constipation. Digestive and Liver Diseas., 2005; 37(11): 838-849.

30. MACHADO WM, CAPELARI SM. Avaliação da eficácia e do grau de adesão ao uso prolongado de fibra dietética no tratamento da constipação intestinal funcional. Rev. Nutr., 2010; 23(2): 231-238.

31. MACHADO WM. Constipação Intestinal. Rev. Bras. de Medicina. 2010; 87-94.

32. MAFFEI HVL, MOREIRA FL, KISSIMOTO M et al. História Clínica e alimentar de crianças atendidas em ambulatório de gastroenterologia pediátrica com constipação intestinal crônica funcional e suas possíveis complicações. J Pediatr, 1994;70:280-6.

33. MAHAN LK, ESCOTT-STUMP S, RAYMOND JL. $13^{\circ}$ ed. Krause: Alimentos, nutrição e dietoterapia.. Rio de Janeiro: Elsevier, 2012.

34. MARTEAU P, CUILLERIER E, MEANCE $S$ et al. Bifidobacteriumanimalis strain DN-173 010 shortens the colonic transit time in healthy women: a double-blind, randomized, controlled study. Aliment Pharmacol Ther., 2002; 16: 587-593.

35. MARTINEZ-COSTA C, ORTUÑO PMJ, PONCE AB, et al. Estreñimiento funcional: estudo prospectivo y respuesta al tratamiento. Pediatr (Barc). 2005;63(5):418-25.

36. MELLO CS, FREITAS KC, TAHAN S et al. Consumo de fibra alimentar por crianças e adolescentes com constipação crônica: influência da mãe ou cuidadora e relação com excesso de peso. Rev. Paul. Pediatr., 2010;28(2): 188-93.

37. MIRA GS, GRAF H, CÂNDIDO LMB.Visão retrospectiva em fibras alimentares com ênfase em beta-glucanas no tratamento do diabetes. Brazilian Journal of Pharmaceutical Sciences, 2009; 45(1):12-20.

38. MONTEIRO JP, CAMELO JÚNIOR JS. Caminhos da nutrição e terapia nutricional: Da concepção à adolescência. Rio de Janeiro: Koogan, 2011. 
39. MORAES JG. Constipação intestinal crônica funcional em crianças: alimentação e ocorrência de bifidobacterium e lactobacillus na microbiota fecal. [Dissertação]. Recife: Universidade Federal de Pernambuco, 2014.

40. MOROTI C, MAGRI LFS, DE SOUZA JCB et al. Potencial da Utilização de Alimentos Probióticos, Prebióticos e Simbióticos na Redução de Colesterol Sanguíneo e Glicemia. Unopar Cient., 2009; 1(3): 63-67.

41. MORRIS DH. Linaza: Una recopilación sobre sus efectosenlasalud y nutrición. $4^{\circ}$ ed. Canadá: Winnipeg, 2007.

42. OSORIO MM, FARIAS JUNIOR G. Padrão alimentar de crianças menores de cinco anos. Revista de Nutrição, $2005 ; 18(6): 793-802$.

43. PAPATHANASOPOULOS A, CAMILLERI M. Dietary fiber supplements: effects in obesity and metabolic syndrome and relationship to gastrointestinal functions. Gastroenterology, 2010; 138(1): 65-72.

44. PAULA LD, MARQUES CF, CHAULD SG. Efeitos produzidos pela ingestão de fibras alimentares: solúveis e insolúveis em camundongos. Rev. Ciência et Praxis, 2009; 2(3): 55-60.

45. PAYNE AN, CHASSARD C, BANZ Y et al. The composition and metabolic activity of child gut microbiota demonstrate differential adaptation to varied nutrient loads in an in vitro model of colonic fermentation. FEMSMicrobiology Ecology, 2012; 80(3): 608-23.

46. PEREIRA VP, MEDEIROS LCS, SPERIDIÃO PGL et al. Percepção das mães sobre a importância das práticas alimentares no tratamento da constipação crônica funcional. Rev. Paul. Pediatr., 2009; 27(1): 33-9.

47. SANDRI R, BERNARDI JR, SIVIERO J. Consumo de fibras e líquidos em mulheres participantes de uma Universidade da Terceira Idade no Sul do Brasil. BCEH, 2012; 9(2): 213-225.

48. SANT'ANNA MSL, FERREIRA CLLF. Prevalência de constipação intestinal no município de Viçosa-MG. Rev. Nutrição Brasil, 2016; 15(1): 11-14.

49. SCHRÖEDER JM, SANTOS P. Efeitos do uso crônico da semente de linhaça (LinumUsitatissimum) sobre a função intestinal e variação do peso corporal em mulheres. Rev. de divulgação científica (ÁGORA), 2009; 1(2): 656-661.

50. SCOTT-STUMP S. Nutriçao relacionada ao diagnóstico e tratamento. $6^{\circ}$ ed. Barueri-SP: Manole, 2011.

51. SCOTT-STUMP S. Nutriçao relacionada ao diagnóstico e tratamento. $6^{\circ}$ ed. Barueri-SP: Manole, 2011.

52. SHEN Q, TUOHY KM, GIBSON GR et al. In vitro measurement of the impact of human milk oligosaccharides on the faecal microbiota of weaned formula-fed infants compared to a mixture of prebiotic fructooligosaccharides and galactooligosaccharides. Letters in Applied Microbiology, 2011; 52 (4): 337-43.

53. SPERIDIÃO PDGL, TAHAN S, FAGUNDES-NETO U, et al. Dietary fiber, energy intake and nutritional status during the treatment of children with chronic constipation. Braz J Med Biol Res., 2003; 36(6):753-9.

54. STEFE CA, ALVES MAR, RIBEIRO RL. Probióticos, prebióticos e simbióticos-artigo de revisão. Saúde \& Ambiente em revista, 2008; 3(1):16-33.

55. THARNER A, JANSEN PW, KIEFTE-DE JONG JC, et al. Bidirectional associations between fussy eating and functional constipation in preschool children. J Pediatr., 2015; 166(1):91-6.

56. TRISÓGLIO C, MARCHI CMG, TORRES US et al. Prevalência de Constipação Intestinal entre Estudantes de Medicina de uma Instituição no Noroeste Paulista. Rev. Brás Coloproct. 2010; 30(2): 203-209.

57. VITOLO MR. Práticas alimentares na infância. In: VITOLO, M. R. Nutrição da gestação ao envelhecimento. Rio de Janeiro: Rubio, 2007, p. 215-242.

58. YANG SD, CHIANG IN, LIN CD et al. Advances in non-surgical treatments for urinary tract infections in children. World Journal of Urology, 2012; 30(1): 69-75.

59. CUPERSMID L, FRAGA APR, ABREU ES et al. Linhaça: Composição Química e Efeitos Biológicos. E-Scientia, $2012 ; 5(2): 33-40$.

60. MOROTI C, MAGRI LFS, SOUZA JCB et al. Potencial da utilização de alimentos probióticos, prebióticos e simbióticos na redução do colesterol sanguíneo e glicemia. Ciênc. Biol. Saúde, 2009; 11(4):63-67. 